\title{
Emergency hernia repair in the elderly: multivariate analysis of morbidity and mortality from an Italian registry
}

\author{
M. Ceresoli ${ }^{1}$ (I) F. Carissimi ${ }^{1}$ - A. Nigro ${ }^{1}$ - P. Fransvea ${ }^{2} \cdot$ L. Lepre $^{3} \cdot$ M. Braga ${ }^{1} \cdot$ G. Costa ${ }^{4}$ on behalf of List of Elderly Risk \\ Assessment and Surgical Outcome (ERASO) Collaborative Study Group endorsed by SICUT, ACOI, SICG, SICE, and \\ Italian Chapter of WSES
}

Received: 3 May 2020 / Accepted: 16 July 2020 / Published online: 31 July 2020

(c) The Author(s) 2020

\begin{abstract}
Purpose The incidence of inguinal hernia is higher in elderly because of aging-related diseases like prostatism, bronchitis, collagen laxity. A conservative management is common in elderly to reduce surgery-related risks, however watchful waiting can expose to obstruction and strangulation. The aim of the present study was to assess the impact of emergency surgery in a large series of elderly with complicated groin hernia and to identify the independent risk factors for postoperative morbidity and mortality. The predictive performance of prognostic risk scores has been also assessed.

Methods This is a prospective observational study carried out between January 2017 and June 2018 in elderly patients who underwent emergency surgery for complicated hernia in 38 Italian hospitals. Pre-operative, surgical and postoperative data were recorded for each patient. ASA score, Charlson's comorbidity index, P-POSSUM and CR-POSSUM were assessed.

Results 259 patients were recruited, mean age was 80 years. A direct repair without mesh was performed in $62(23.9 \%)$ patients. Explorative laparotomy was performed in $56(21.6 \%)$ patients and bowel resection was necessary in $44(17 \%)$. Mortality occurred in seven (2.8\%) patients. Fifty-five (21.2\%) patients developed complications, 12 of whom had a major one. At univariate and multivariate analyses, Charlson's comorbidity index $\geq 6$, altered mental status, and need for laparotomy were associated with major complications and mortality

Conclusion Emergency surgery for complicated hernia is burdened by high morbidity and mortality in elderly patients. Preoperative comorbidity played a pivotal role in predicting complications and mortality and therefore Charlson's comorbidity index could be adopted to select patients for elective operation
\end{abstract}

Keywords Groin hernia · Incarcerated hernia · Elderly · Postoperative complications · Emergency surgery · Charlson's comorbidity index

\section{Introduction}

The members of "The ERASO Study Group" are listed in acknowledgements.

Electronic supplementary material The online version of this article (https://doi.org/10.1007/s10029-020-02269-5) contains supplementary material, which is available to authorized users.

M. Ceresoli

Marco.ceresoli@libero.it

1 General and Emergency Surgery Department, School of Medicine and Surgery, University of Milano-Bicocca, via Pergolesi 33, 20900 Monza, Italy

2 UOC Chirurgia D’Urgenza E del Trauma, Fondazione Policlinico Universitario A. Gemelli IRCCS, Università Cattolica del Sacro Cuore, Roma, Italy
Inguinal and femoral hernias are very common clinical situations worldwide with estimated prevalence of $27-43 \%$ in men and 3-6\% in women [1]. Despite groin hernia is widespread in all age groups of population, its incidence is higher

3 UOC Chirurgia Generale, Ospedale Santo Spirito in Sassia, ASL Roma 1, Roma, Italy

4 Surgical and Medical Department of Translational Medicine, Sant'Andrea Teaching Hospital, Sapienza University of Roma, Roma, Italy 
in elderly [2]. Conditions frequently associated to advanced age, such as constipation, prostatism, frequent coughing due to respiratory diseases and weakness of the abdominal wall, play an important role in the development and evolution of abdominal wall hernias $[2,3]$.

Groin hernias can progress to incarceration and strangulation which constitute a common surgical emergency. The estimated risk of an inguinal hernia becoming incarcerated is $4.5 \%$ after 2 years and the complication risk is higher in femoral hernia with a $22 \%$ cumulative probability at 3 months and $45 \%$ at 21 months [4].

Regardless of age and frailty European Hernia Society Guidelines recommend surgery in case of symptomatic inguinal hernia; whereas if patients do not complain of symptoms the indication to surgical repair is debated, being a watchful approach an option [3]. Although elective surgery repair is performed safely with minimal morbidity $[5$, 6] conservative treatment is sometimes preferred in elderly due to comorbidities. On the other hand, the natural history of a conservatively managed groin hernia is size increasing due to continuous action of intra-abdominal pressure and progressive abdominal wall laxity [3]. This exposes patients to an increasing risk of bowel obstruction and strangulation requiring emergency surgery with consequent risk of laparotomy and bowel resection [7]. The balance between the risks of elective surgery versus the risks of a watchful approach is still a matter of debate in absence of specific recommendations for elderly.

The aim of the present study was to assess the impact of emergency surgery in a large series of elderly patients with complicated groin hernia and to identify independent risk factors for postoperative morbidity and mortality. The predictive performance of prognostic risk scores has also been assessed.

\section{Methods}

The present study analyzed data from the Frailty and Emergency Surgery Study (FRAILESEL) database [8]; FRAILESEL is a prospective observational project that collected data in consecutive elderly patients who underwent emergency surgery in 38 Italian hospitals. The Study protocol was approved by the Ethics Committee of Sapienza University of Rome and of all participating centers and was registered on clinicaltrials.gov (ClinicalTrials.gov identifier: NCT02825082). All patients who underwent emergency surgery for incarcerated inguinal or femoral hernia between January 2017 and June 2018 were included in the present study. For each patient the following data were recorded: age, sex, BMI, comorbidities, American Society of Anesthesiologist (ASA) score, preoperative hemodynamic status, type of incarcerated hernia (inguinal or femoral), surgical technique, need for explorative laparotomy and bowel resection. For each patient, the Charlson's comorbidity index [9, 10], the predicted morbidity and mortality risks according to the P-POSSUM and the CR-POSSUM models [11, 12] were also calculated.

All postoperative complications and reoperations that occurred during hospitalization or within 30 days after discharge were registered and graded according to the ClavienDindo classification [13].

Continuous variables were expressed as mean (SD) or median (IQR) as appropriate; categorical data were showed as proportion and percentages. Five different variables were selected as outcomes: explorative laparotomy, abdominal viscera resection, complications, major complications (Clavien-Dindo $\geq \mathrm{IIIb}$ ), and mortality. Univariate analysis was carried out with the chi square test and Mann-Whitney $U$ test; variables significantly associated with the outcomes were inserted in a multivariate model with the logistic regression method; multivariate analysis was not computed in case of number of events $<10$.The ASA scores and the Charlson's comorbidity index were analyzed with the ROC curves method in order to choose a cut-off for complications, major complications and mortality. ASA score (both as categorical and with the cut-off chosen with the ROC method), the Charlson's comorbidity index (both as continuous, categorical the cut-off chosen with the ROC method), the predicted risk of morbidity and mortality with the P-POSSUM and CR-POSSUM models and the length of stay were compared among patients with or without the selected outcomes (morbidity and mortality) with the appropriate test. Statistics were calculated with SPSS 25 IBM Corp. Released 2017. IBM SPSS Statistics for Windows, Version 25.0. Armonk, NY: IBM Corp.).

\section{Results}

A total of 259 consecutive patients operated for complicated inguinal or femoral hernia were included in the analysis.

Table 1 reports patients' characteristics in detail. Mean age was $80( \pm 8)$ years and $58 \%$ patients were men. Common comorbidities were hypertension $(65 \%)$, chronic heart disease (28\%), arrhythmia (34\%), and COPD (18\%) while $20 \%$ of patients were in therapy with oral anticoagulants. Patients with inguinal hernia were similar to those with femoral hernia in terms of comorbidity and pre-operative characteristics; as expected female sex was more common in femoral hernia (84\% vs. $23 \%, p<0.001)$.

Table 2 shows surgical data and outcomes. One hundred and eighty $(69.5 \%)$ patients were operated for inguinal hernia and 79 (30.5\%) for femoral hernia. Laparoscopic surgery was carried out in $10(12.66 \%)$ patients. A mesh repair was performed in $91 \%$ of patients with inguinal hernia and 
Table 1 Patients characteristics

\begin{tabular}{|c|c|c|c|c|}
\hline & Mean (SD) & Median (IQR) & $N$ & $\%$ \\
\hline Number of patients & & & 259 & \\
\hline Age & $79.70(8.37)$ & $79(73-87)$ & & \\
\hline \multicolumn{5}{|l|}{ Age class } \\
\hline $65-70$ & & & 41 & $15.1 \%$ \\
\hline $71-75$ & & & 49 & $18.3 \%$ \\
\hline $76-80$ & & & 51 & $19.7 \%$ \\
\hline $81-85$ & & & 39 & $15.1 \%$ \\
\hline $86-90$ & & & 51 & $19.7 \%$ \\
\hline$>90$ & & & 28 & $10.8 \%$ \\
\hline \multicolumn{5}{|l|}{ Sex } \\
\hline Female & & & 109 & $42.1 \%$ \\
\hline Male & & & 150 & $57.9 \%$ \\
\hline BMI & $25.36(5.67)$ & $24.74(22-27)$ & & \\
\hline Mental status impairment & & & 14 & $6.3 \%$ \\
\hline Hypotension $(\mathrm{SBP}<90)$ & & & 3 & $1.2 \%$ \\
\hline Tachycardia (HR > 100) & & & 14 & $5.4 \%$ \\
\hline \multicolumn{5}{|l|}{ Comorbidity } \\
\hline Atrial Fibrillation/arrhythmia & & & 96 & $37.1 \%$ \\
\hline Ischemic heart disease & & & 8 & $3.1 \%$ \\
\hline Chronic heart disease & & & 73 & $28.2 \%$ \\
\hline Arterial hypertension & & & 168 & $64.9 \%$ \\
\hline Peripheral artery disease & & & 37 & $14.3 \%$ \\
\hline Cerebrovascular disease & & & 38 & $14.7 \%$ \\
\hline Oral Anticoagulants & & & 52 & $20.1 \%$ \\
\hline COPD & & & 48 & $18.5 \%$ \\
\hline Metastatic Cancer & & & 9 & $3.5 \%$ \\
\hline Cancer without metastasis & & & 23 & $8.9 \%$ \\
\hline Leukemia/lymphoma & & & 7 & $2.7 \%$ \\
\hline Hepatic disease & & & 10 & $3.9 \%$ \\
\hline Kidney disease & & & 22 & $8.5 \%$ \\
\hline Diabetes & & & 32 & $16.2 \%$ \\
\hline Peptic ulcer & & & 6 & $2.3 \%$ \\
\hline Connective tissue disease & & & 10 & $3.9 \%$ \\
\hline Steroids/immunosuppressive & & & 14 & $5.4 \%$ \\
\hline Emiplegia & & & 10 & $3.9 \%$ \\
\hline Demenza & & & 27 & $10.4 \%$ \\
\hline \multicolumn{5}{|l|}{ ASA score } \\
\hline 1 & & & 12 & $4.7 \%$ \\
\hline 2 & & & 88 & $34.8 \%$ \\
\hline 3 & & & 132 & $52.2 \%$ \\
\hline 4 & & & 20 & $7.9 \%$ \\
\hline 5 & & & 1 & $0.4 \%$ \\
\hline Charlson comorbidity index & $4.97(2.28)$ & $4(3-6)$ & & \\
\hline \multicolumn{5}{|l|}{ Charlson comorbidity index } \\
\hline$<6$ & & & 176 & $68 \%$ \\
\hline$\geq 6$ & & & 83 & $32 \%$ \\
\hline \multicolumn{5}{|l|}{ Charlson comorbidity index } \\
\hline $0-1$ & & & 0 & $0 \%$ \\
\hline $2-3$ & & & 71 & $27 \%$ \\
\hline $4-5$ & & & 105 & $40.5 \%$ \\
\hline $6-7$ & & & 46 & $17.8 \%$ \\
\hline
\end{tabular}


Table 1 (continued)

\begin{tabular}{lllrr}
\hline & Mean (SD) & Median (IQR) & \multicolumn{1}{c}{$N$} & $\%$ \\
\hline $8-9$ & & & 28 & $10.8 \%$ \\
$10-11$ & & & 5 & $1.9 \%$ \\
$12-13$ & & & 2 & $0.8 \%$ \\
$14-15$ & & & $0.4 \%$ \\
$16-17$ & & & 1 & $0.4 \%$ \\
Predicted mortality risk (PPOSSUM) & $7.81(12.46)$ & $3.60(1.5-8.1)$ & & \\
Predicted morbidity risk (PPOSSUM) & $50.68(24.11)$ & $49(29-71)$ & & \\
Predicted mortality risk (CR-POSSUM) & $6.88(7.34)$ & $5.10(1.9-9)$ & & \\
\hline
\end{tabular}

Table 2 Surgery data and outcomes

\begin{tabular}{|c|c|c|c|c|}
\hline & Mean (SD) & Median (IQR) & $n(\%)$ & $\%$ \\
\hline Time to surgery (days) & $0.58(1.49)$ & $0(0-1)$ & & \\
\hline \multicolumn{5}{|l|}{ Kind of hernia } \\
\hline Inguinal & & & 180 & $69.11 \%$ \\
\hline Femoral & & & 79 & $30.12 \%$ \\
\hline \multicolumn{5}{|l|}{ Inguinal hernia } \\
\hline Direct repair & & & 15 & $8.33 \%$ \\
\hline Mesh & & & 165 & $91.67 \%$ \\
\hline \multicolumn{5}{|l|}{ Femoral hernia } \\
\hline Direct repair & & & 47 & $59.49 \%$ \\
\hline Mesh & & & 32 & $40.51 \%$ \\
\hline Laparoscopic repair & & & 10 & $12.66 \%$ \\
\hline Explorative laparotomy/laparoscopy & & & 56 & $21.62 \%$ \\
\hline \multicolumn{5}{|l|}{ Intestinal resection } \\
\hline No & & & 215 & $83.01 \%$ \\
\hline Colon & & & 2 & $0.77 \%$ \\
\hline Ileum & & & 41 & $15.83 \%$ \\
\hline Ileum-cecum & & & 1 & $0.39 \%$ \\
\hline Length of stay & $5.17(4.02)$ & $4.00(2.00-7.00)$ & & \\
\hline Reintervention & & & 3 & $1.16 \%$ \\
\hline Major complications & & & 12 & $4.63 \%$ \\
\hline Complications & & & 55 & $21.24 \%$ \\
\hline Perforation & & & 2 & $0.77 \%$ \\
\hline Occlusion & & & 5 & $1.93 \%$ \\
\hline Pneumonia & & & 8 & $3.09 \%$ \\
\hline Acute renal failure & & & 4 & $1.54 \%$ \\
\hline Bleeding & & & 5 & $1.93 \%$ \\
\hline Stroke & & & 2 & $0.77 \%$ \\
\hline Acute myocardial infarction/heart failure & & & 3 & $1.16 \%$ \\
\hline Arrhythmia & & & 5 & $1.93 \%$ \\
\hline SSI & & & 6 & $2.32 \%$ \\
\hline Mortality & & & 7 & $2.70 \%$ \\
\hline
\end{tabular}

in $41 \%$ with femoral hernia. At univariate analysis, factors related to the mesh placement were increasing BMI (as a continuous variable $)(\mathrm{OR}=1.147$; CI 95\% $=1.040-1.264)$; male gender $(\mathrm{OR}=5.501$; CI 95\% = 2.922-10.35); femoral hernia $(\mathrm{OR}=0.062$; CI $95 \%=0.031-0.124)$, need for explorative laparotomy $(\mathrm{OR}=0.379 ; \mathrm{CI} 95 \%=0.200-0.718)$ and bowel resection $(\mathrm{OR}=0.230$; CI 95\% $=0.119-0.446)$. At multivariate analysis only femoral hernia maintained an independent association with mesh $(\mathrm{OR}=0.64$; CI $95 \%=0.021-0.199)$.

An explorative laparotomy was necessary in 56 $(21.6 \%)$ patients and $44(17.0 \%)$ of them had a bowel 
resection. At multivariate analysis, significant risk factors were ASA score $>2$ for laparotomy and femoral hernia for bowel resection (Table 3 ).

Post-operative outcomes are reported in Table 2. Overall morbidity was $21.2 \%$. Major complications occurred in $12(4.6 \%)$ patients and mortality in seven $(2.8 \%)$ patients. Three patients died for sepsis, one for heart failure, acute cardiac ischemia, stroke, and hemorrhage. Mean length of stay was significantly longer in patients with complications than in uneventful (8.3 vs. $4.3, p<0.001)$ (Table 4$)$.

The multivariate analysis demonstrated that preoperative conditions, such as heart and lung dysfunctions and Charlson's comorbidity index $\geq 6$, were independently associated with major complications and mortality (Table 5).

\section{Prognostic scores}

The predicted risk according to the P-POSSUM model was $50 \%( \pm 24)$ for morbidity and $7.81 \%( \pm 12)$ for mortality. The CR-POSSUM model prediction mortality was $6.88 \%$ $( \pm 7)$.

With the ROC curves method were individuated two cutoff for the ASA score (cut-off three) and Charlson's comorbidity index (cut-off six) (see supplementary materials).

Major morbidity was $5.2 \%$, in patients with ASA score $\geq 3$ compared with $3.8 \%$ in patients with ASA $<3(p=0.584)$. In patients with Charlson's comorbidity index $\geq 6$ major morbidity was $8.4 \%$ compared with $2.8 \%$ in patients with index $<6(p<0.045)$. Mortality with ASA score $\geq 3$ was 4.1 , compared with $1 \%$ in patients with ASA $<3(p=0.141)$. In patients with Charlson's comorbidity index $\geq 6$ mortality was $8 \%$ compared with $0 \%$ in patients with index $<6(p<0.001)$. Results are shown in detail in Table 4 and Fig. 1.

Table 3 Univariate and multivariate analysis of factor associated to laparotomy and resection

\begin{tabular}{|c|c|c|c|c|c|c|c|c|}
\hline & \multicolumn{4}{|l|}{ laparotomy } & \multicolumn{4}{|l|}{ Resection } \\
\hline & $\begin{array}{l}\text { OR }(95 \% \mathrm{CI}) \text { uni- } \\
\text { variate }\end{array}$ & $p$ & $\begin{array}{l}\text { OR }(95 \% \mathrm{CI}) \text { multi- } \\
\text { variate }\end{array}$ & $p$ & $\begin{array}{l}\text { OR }(95 \% \mathrm{CI}) \text { uni- } \\
\text { variate }\end{array}$ & $p$ & $\begin{array}{l}\text { OR }(95 \% \mathrm{CI}) \text { multi- } \\
\text { variate }\end{array}$ & $p$ \\
\hline Age & $1.006(0.971-1.042)$ & 0.736 & & & $0.984(0.948-1.022)$ & 0.399 & & \\
\hline BMI & $0.974(0.906-1.048)$ & 0.482 & & & $0.941(0.859-1.031)$ & 0.193 & & \\
\hline Male sex & $0.606(0.334-1.098)$ & 0.097 & & & $0.784(0.42-1.464)$ & 0.445 & & \\
\hline $\mathrm{ASA} \geq 3$ & $3.16(1.57-6.33)$ & 0.001 & $1.876(1.165-3.021)$ & $\mathbf{0 . 0 1}$ & $1.94(0.991-3.831)$ & 0.051 & & \\
\hline Charlson $\geq 6$ & $1.66(0.901-3.065)$ & 0.102 & & & $1.44(0.758-2.754)$ & 0.262 & & \\
\hline $\begin{array}{l}\text { Femoral Hernia } \\
\text { (inguinal ref) }\end{array}$ & $1.830(0.989-3.384)$ & 0.052 & & & $2.187(1.153-4.147)$ & 0.015 & $2.275(1.190-4.348)$ & 0.013 \\
\hline Arrhythmia & $1.241(0.678-2.227)$ & 0.483 & & & $1.216(0.644-2.296)$ & 0.546 & & \\
\hline $\begin{array}{l}\text { Myocardial infarc- } \\
\text { tion }\end{array}$ & $1.216(0.239-6.196)$ & 0.814 & & & $1.447(0.283-7.395)$ & 0.656 & & \\
\hline Chronic heart disease & $\begin{array}{l}3.882(1.082- \\
13.925)\end{array}$ & 0.026 & $2.260(0.577-8.846)$ & 0.242 & $\begin{array}{l}3.022(0.819- \\
11.153)\end{array}$ & 0.083 & & \\
\hline Hypertension & $0.858(0.464-1.586)$ & 0.625 & & & $0.652(0.346-1.231)$ & 0.186 & & \\
\hline $\begin{array}{l}\text { Cerebrovascular } \\
\text { disease }\end{array}$ & $1.359(0.616-2.999)$ & 0.447 & & & $0.776(0.305-1.974)$ & 0.594 & & \\
\hline Oral anticoagulants & $1.11(0.538-2.297)$ & 0.776 & & & $1.193(0.562-2.533)$ & 0.645 & & \\
\hline $\begin{array}{l}\text { Chronic lung dis- } \\
\text { eases }\end{array}$ & $1.657(0.816-3.364)$ & 0.159 & & & $1.568(0.745-3.279)$ & 0.233 & & \\
\hline $\begin{array}{l}\text { Metastatic solid } \\
\text { tumors }\end{array}$ & $1.858(0.45-7.679)$ & 0.385 & & & $\begin{array}{l}\text { 3.644 (1.041- } \\
14.112)\end{array}$ & 0.047 & $4.008(1.029-16.24)$ & 0.045 \\
\hline $\begin{array}{l}\text { Non-metastatic solid } \\
\text { tumors }\end{array}$ & $0.745(0.243-2.286)$ & 0.606 & & & $0.62(0.177-2.1734)$ & 0.451 & & \\
\hline Liver disease & $0.903(0.186-4.376)$ & 0.899 & & & $1.891(0.471-7.592)$ & 0.362 & & \\
\hline Kidney disease & $1.073(0.378-3.047)$ & 0.895 & & & $0.948(0.306-2.938)$ & 0.926 & & \\
\hline Diabetes & $0.440(0.164-1.178)$ & 0.095 & & & $0.402(0.136-1.186)$ & 0.089 & & \\
\hline $\begin{array}{l}\text { Steroids/immunosop- } \\
\text { pressors }\end{array}$ & $1.485(0.447-4.926)$ & 0.516 & & & $2.538(0.811-7.942)$ & 0.099 & & \\
\hline Dementia & $2.857(1.241-6.577)$ & 0.011 & $2.051(0.841-5.001)$ & 0.114 & $1.961(0.804-4.787)$ & 0.133 & & \\
\hline Leukemia/lymphoma & $0.597(0.07-5.064)$ & 0.633 & & & $1.745(0.328-9.270)$ & 0.509 & & \\
\hline
\end{tabular}

Bold indicate depicted significative results 


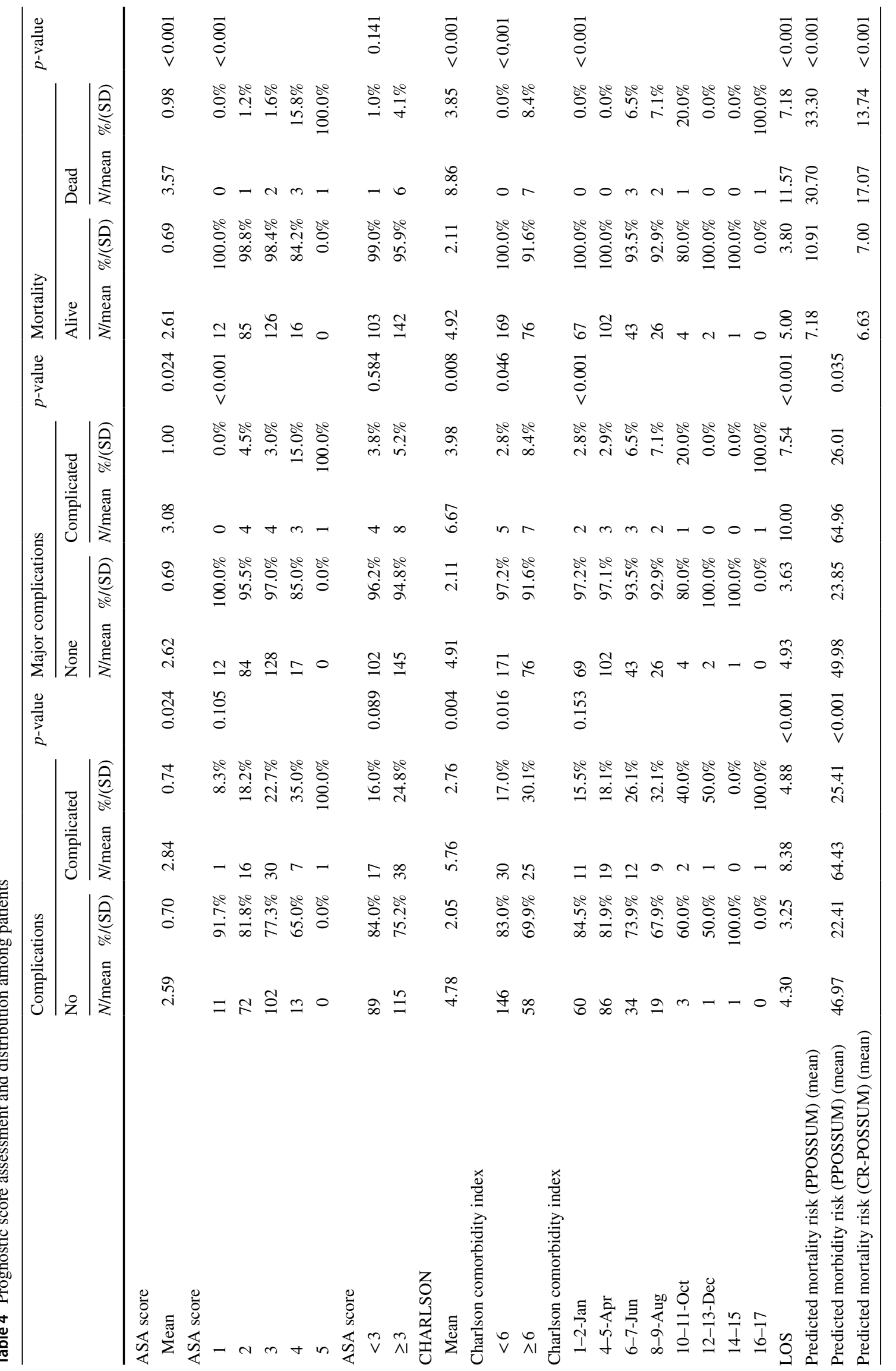


Table 5 Univariate and multivariate analysis for complications and mortality

\begin{tabular}{|c|c|c|c|c|c|c|c|c|}
\hline & \multicolumn{4}{|l|}{ Complication } & \multicolumn{2}{|l|}{ Major complication } & \multicolumn{2}{|l|}{ Mortality } \\
\hline & $\begin{array}{l}\text { OR }(95 \% \mathrm{CI}) \\
\text { univariate }\end{array}$ & $p$ & $\begin{array}{l}\text { OR }(95 \% \mathrm{CI}) \\
\text { multivariate }\end{array}$ & $p$ & $\begin{array}{l}\text { OR }(95 \% \mathrm{CI}) \text { uni- } \\
\text { variate }\end{array}$ & $p$ & $\begin{array}{l}\text { OR }(95 \% \mathrm{CI}) \text { uni- } \\
\text { variate }\end{array}$ & $p$ \\
\hline Age & $\begin{array}{l}1.010(0.974- \\
1.046)\end{array}$ & 0.594 & & & $\begin{array}{l}0.959(0.892- \\
1.031)\end{array}$ & 0.256 & $\begin{array}{l}1.019(0.931- \\
1.115)\end{array}$ & 0.69 \\
\hline BMI & $\begin{array}{l}0.968(0.902- \\
1.032)\end{array}$ & 0.364 & & & $\begin{array}{l}0.924(0.784- \\
1.089)\end{array}$ & 0.347 & $\begin{array}{l}0.842(0.687- \\
1.031)\end{array}$ & 0.096 \\
\hline hypotension & $\begin{array}{l}7.843(0.678- \\
88.20)\end{array}$ & 0.051 & & & $53.74(4.45-649)$ & $<0.001$ & $120(8.95-1600)$ & $<0.001$ \\
\hline Tachycardia & $4.217(1.41-12.74)$ & 0.006 & $\begin{array}{l}2.889(0.673- \\
12.40)\end{array}$ & 0.154 & 7.909 (1.84-33.98) & 0.001 & 23.1 (4.13-129) & $<0.001$ \\
\hline Mental impairment & $\begin{array}{l}3.837(1.277- \\
11.529)\end{array}$ & 0.011 & $\begin{array}{l}2.502(0.626- \\
9.993)\end{array}$ & 0.194 & $10(2.57-38.88)$ & $<0.001$ & $\begin{array}{c}26.4(5.192- \\
134.226)\end{array}$ & $<0.001$ \\
\hline Male Sex & $\begin{array}{l}0.635(0.349- \\
1.155)\end{array}$ & 0.135 & & & $\begin{array}{l}1.018(0.314- \\
3.297)\end{array}$ & 0.976 & $\begin{array}{l}1.875(0.357- \\
9.854)\end{array}$ & 0.451 \\
\hline $\mathrm{ASA} \geq 3$ & $\begin{array}{l}1.730(0.916- \\
3.265)\end{array}$ & 0.089 & & & $\begin{array}{l}1.407(0.413- \\
4.797)\end{array}$ & 0.584 & $\begin{array}{l}4.352(0.516- \\
36.70)\end{array}$ & 0.141 \\
\hline Charlson $\geq 6$ & $2.09(1.138-3.867)$ & 0.016 & $\begin{array}{l}1.105(0.624- \\
1.956)\end{array}$ & 0.732 & $3.150(1.03-10.24)$ & 0.046 & - & $<0.001$ \\
\hline Crural hernia & $\begin{array}{l}1.404(0.750- \\
2.630)\end{array}$ & 0.287 & & & $\begin{array}{l}1.147(0.335- \\
3.925)\end{array}$ & 0.827 & $\begin{array}{l}0.889(0.169- \\
4.687)\end{array}$ & 0.89 \\
\hline Laparotomy & $\begin{array}{l}4.161(2.166- \\
7.995)\end{array}$ & $<0.001$ & $\begin{array}{l}6.607(2.905- \\
15.03)\end{array}$ & $<0.001$ & $\begin{array}{l}5.657(1.722- \\
18.586)\end{array}$ & 0.002 & $5.2(1.127-23.987)$ & 0.02 \\
\hline Prosthesis & $0.795(0.433-1.46)$ & 0.46 & & & $\begin{array}{l}1.008(0.285- \\
3.571)\end{array}$ & 0.99 & $\begin{array}{l}1.034(0.205- \\
5.223)\end{array}$ & 0.968 \\
\hline Bowel resection & $\begin{array}{l}3.448(1.755- \\
6.776)\end{array}$ & $<0.001$ & $\begin{array}{l}0.721(0.193- \\
3.165)\end{array}$ & 0.728 & $\begin{array}{l}6.833(2.069- \\
22.567)\end{array}$ & $<0.001$ & $\begin{array}{l}6.264(1.353- \\
29.005)\end{array}$ & 0.008 \\
\hline Arrhythmia & $\begin{array}{l}2.074(1.134- \\
3.792)\end{array}$ & 0.017 & $\begin{array}{l}2.813(1.317- \\
6.008)\end{array}$ & 0.008 & $\begin{array}{l}1.224(.0378- \\
3.971)\end{array}$ & 0.735 & $\begin{array}{l}1.247(0.273- \\
5.698)\end{array}$ & 0.775 \\
\hline $\begin{array}{l}\text { Ischemic heart } \\
\text { disease }\end{array}$ & $\begin{array}{l}2.296(0.531- \\
9.922)\end{array}$ & 0.253 & & & $\begin{array}{c}8.003(1.437- \\
44.897)\end{array}$ & 0.005 & $\begin{array}{l}15.933(2.558- \\
99.23)\end{array}$ & $<0.001$ \\
\hline $\begin{array}{l}\text { Chronic heart } \\
\text { disease }\end{array}$ & $\begin{array}{l}2.588(0.704- \\
9.516)\end{array}$ & 0.139 & & & $\begin{array}{c}11.429(2.531- \\
51.597)\end{array}$ & $<0.001$ & $\begin{array}{l}11.850(1.898- \\
70.605)\end{array}$ & 0.001 \\
\hline Hypertension & $\begin{array}{l}1.739(0.890- \\
3.397)\end{array}$ & 0.103 & & & $\begin{array}{l}2.767(0.593- \\
12.91)\end{array}$ & 0.178 & $\begin{array}{l}3.188(0.378- \\
26.91)\end{array}$ & 0.261 \\
\hline $\begin{array}{l}\text { cerebrovascular } \\
\text { disease }\end{array}$ & $\begin{array}{l}1.181(0.522- \\
2.668)\end{array}$ & 0.689 & & & $\begin{array}{l}1.172(0.247- \\
5.572)\end{array}$ & 0.841 & $\begin{array}{l}2.322(0.434- \\
12.42)\end{array}$ & 0.312 \\
\hline Oral anticoagulants & $\begin{array}{l}1.915(0.966- \\
3.795)\end{array}$ & 0.06 & & & 3.04 (0.924-9.999) & 0.056 & $\begin{array}{l}5.617(1.216- \\
25.95)\end{array}$ & 0.014 \\
\hline COPD & $\begin{array}{l}2.205(1.102- \\
4.412)\end{array}$ & 0.023 & $\begin{array}{l}2.505(1.024- \\
6.126)\end{array}$ & 0.044 & $\begin{array}{l}3.389 \text { (1.027- } \\
11.182)\end{array}$ & 0.035 & $\begin{array}{l}3.426(0.74- \\
15.855)\end{array}$ & 0.095 \\
\hline $\begin{array}{l}\text { Metastatic solid } \\
\text { tumors }\end{array}$ & $1.904(0.461-7.87)$ & 0.366 & & & $\begin{array}{l}2.716(0.312- \\
23.666)\end{array}$ & 0.347 & $\begin{array}{l}4.938(0.53- \\
45.972)\end{array}$ & 0.121 \\
\hline $\begin{array}{l}\text { Non-metastatic } \\
\text { solid tumors }\end{array}$ & $\begin{array}{l}1.347(0.504- \\
3.598)\end{array}$ & 0.551 & & & $\begin{array}{c}2.152(0.442- \\
10.478)\end{array}$ & 0.332 & $4.5(0.82-24.692)$ & 0.059 \\
\hline Liver disease & $0.401(0.05-3.237)$ & 0.376 & & & - & 0.477 & - & 0.606 \\
\hline Kidney disease & $1.838(0.71-4.758)$ & 0.205 & & & $\begin{array}{l}2.27(0.465- \\
11.082)\end{array}$ & 0.298 & $4.5(0.82-24.692)$ & 0.59 \\
\hline Diabetes & $\begin{array}{l}1.868(0.895- \\
3.898)\end{array}$ & 0.093 & & & $\begin{array}{l}1.778(0.461- \\
6.862)\end{array}$ & 0.398 & $\begin{array}{l}4.086(0.879- \\
18.98)\end{array}$ & 0.053 \\
\hline $\begin{array}{l}\text { Immunosuppres- } \\
\text { sive drugs }\end{array}$ & 3 (1.004-9.047) & 0.042 & $\begin{array}{c}3.684(0.941- \\
14.42)\end{array}$ & 0.061 & $\begin{array}{l}1.636(0.196- \\
3.659)\end{array}$ & 0.646 & $\begin{array}{l}2.974(0.333- \\
26.562)\end{array}$ & 0.306 \\
\hline Dementia & $\begin{array}{l}2.022(0.853- \\
4.791)\end{array}$ & 0.104 & & & $\begin{array}{l}4.87(1.361- \\
17.421)\end{array}$ & 0.008 & $\begin{array}{l}13.515(2.841- \\
64.297)\end{array}$ & $<0.001$ \\
\hline $\begin{array}{l}\text { Leukemia/lym- } \\
\text { phoma }\end{array}$ & $\begin{array}{l}0.611(0.072- \\
5.185)\end{array}$ & 0.649 & & & $\begin{array}{c}3.652(0.404- \\
33.006)\end{array}$ & 0.218 & $\begin{array}{l}6.639(0.688- \\
64.05)\end{array}$ & 0.06 \\
\hline
\end{tabular}



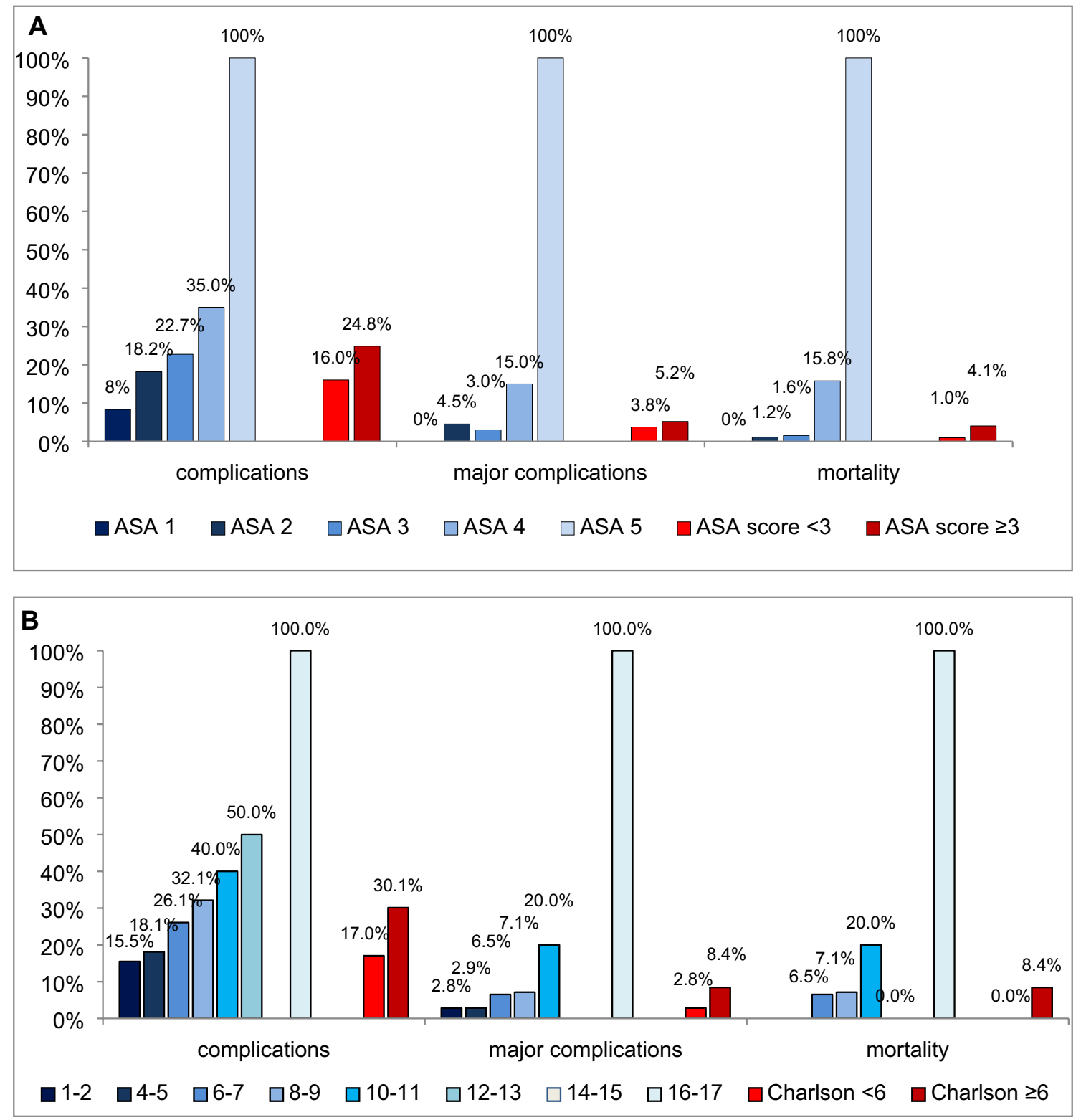

Fig. 1 a complications, major complications and mortality rates among ASA score (a) and Charlson's comorbidity index (b) classes

\section{Discussion}

The present study shows that emergency surgery for complicated hernia is burdened by high morbidity and mortality in elderly patients. Femoral hernia was associated with a higher risk of laparotomy and bowel resection. Heart and lung dysfunction, impaired mental status, and oral anticoagulant therapy were correlated to postoperative complications and mortality.

In current practice, elderly patients presenting with asymptomatic groin hernia are often managed conservatively to avoid the surgery-related risk of complications. A watchful waiting is recognized as an acceptable option for patients with asymptomatic or minimally symptomatic inguinal hernias $[14,15]$. On the other hand, an incarcerated hernia can be sometimes difficult to identify by physical examination [16] and a delayed diagnosis could significantly increase the risk of strangulation. Incarceration and even more strangulation seldom occur, but require mandatory emergency surgery which is burdened by higher mortality and morbidity in elderly when compared to younger patients $[17,18]$. In the emergency setting general anesthesia is usually preferred, whereas local or loco-regional anesthesia is the first option for elective hernia repair, especially in elderly patients with severe comorbidities [19]. 
In the present study the overall postoperative mortality $(2.8 \%)$ was substantially higher than those reported after elective hernia repair in elderly [20]. In the subgroup of patients who had laparotomy and bowel resection mortality was $7.14 \%$, consistent with previous series reporting a mortality increase up to $20 \%$ in case of ischemic herniated bowel resection [20-22]. Overall morbidity was $21 \%$ and major complication rate was $5 \%$, both aligning with the existing literature on emergency surgery for complicated hernia [23], but much higher when compared to elective surgery [24].

At multivariate analysis, impaired mental status, heart and lung dysfunctions, and oral anticoagulant therapy were independently associated to major complications and mortality. Noteworthy, diabetes was not associated with morbidity or mortality in our cohort of patients. Usually, the presence of comorbidities advises physicians to prefer a watchful approach. Despite the present study cannot demonstrate the superiority of an operative approach to groin hernia due to the lack of a control population the indication to perform an elective procedure should be carefully tailored, balancing the risk of hernia incarceration and the risk of postoperative complications, in case of emergency surgery. A particular attention should be reserved to patients with oral anticoagulants that can be safely stopped in proper time in case of elective surgery, but not in emergency setting.

To predict surgical risk in patients undergoing emergency surgery for incarcerated/strangulated groin hernia some common preoperative score have been tested. ASA score and above all Charlson's comorbidity index allowed an easy and rapid stratification of patients at high risk for morbidity and mortality. Conversely, P-POSSUM and CR-POSSUM which has been specifically validated for colorectal and major surgery, failed to predict morbidity and mortality, with a predicted risk overestimation. Therefore, ASA score and Charlson's comorbidity index could be adopted as valid tools for risk stratification in elderly to select candidates for elective hernia repair.

In patients undergoing emergency surgery, the use of mesh to repair hernia is still an open issue because prosthesis could increase the infectious risk [25]. However, in accordance with the EHS guidelines [3], a direct repair without mesh brings a greater risk of recurrence with possible need of redo surgery. According to WSES guidelines [16] a mesh should be used in clean and clean contaminated (CDC class I and II) [13] emergency setting, while the use of mesh should be discouraged in dirty/contaminated surgery which is burdened by an infection rate up to $38 \%$ following bowel resection [26]. In the present study the only independent factor related to direct repair was femoral hernia. An high proportion of patients with femoral hernia in fact did not receive mesh positioning (59.5\%), exposing them to the risk of recurrence; on the contrary a great proportion of patients operated for inguinal hernia had the positioning of a mesh, despite the presence of strangulated/incarcerated viscera and the consequent risk of infection. In our series of elderly patients factors associated with the non-positioning of mesh were explorative laparotomy and bowel resection, both indicating the presence of a contaminated surgical field. Moreover also the age could have played an important role: the lower life expectation of elderly could have mitigated the risk of recurrence linked to the direct repair.

The observational multicentre cohort design without a control population to compare is a limitation of the present study, therefore no clear recommendations could be derived from the present paper; moreover the study was not originally designed specifically for groin hernia and therefore some important information are missing like the timeframe between incarceration and presentation in hospital. However, the prospective data collection and a priori definition of criteria to identify postoperative complications might mitigate this limitation. Moreover, a multicentre study allows better generalization of results than single centre, while the large series of patients allowed excluding confounders by multiple logistic analyses.

In conclusion, emergency surgery for complicated hernia is burdened by high morbidity and mortality in elderly patients. Femoral hernia was associated with a higher risk of laparotomy and bowel resection. Since preoperative comorbidity played a pivotal role in predicting complications and mortality, Charlson's comorbidity index should be adopted as a valid tool for evaluate and select patients for elective operation.

Acknowledgements Open access funding provided by Universit $\tilde{A}$ degli Studi di Milano - Bicocca within the CRUI-CARE Agreement. List of Elderly Risk Assessment and Surgical Outcome (ERASO) Collaborative Study Group endorsed by SICUT, ACOI, SICG, SICE, and Italian Chapter of WSES: F. Agresta, G. Alemanno, G. Anania, M. Antropoli, G. Argenio, J. Atzeni, N. Avenia, A. Azzinnaro, G. Baldazzi, G. Balducci, G. Barbera, G. Bellanova, C. Bergamini, L. Bersigotti, PP. Bianchi, C. Bombardini, G. Borzellino, S. Bozzo, G. Brachini, GM. Buonanno, T. Canini, S. Cardella, G. Carrara, D. Cassini, M. Castriconi, G. Ceccarelli, D. Celi, M. Ceresoli, M. Chiappetta, M. Chiarugi, N. Cillara, F. Cimino, L. Cobuccio, G. Cocorullo, E. Colangelo, G. Costa, A. Crucitti, P. DallaCaneva, M. De Luca, A. de Manzoni Garberini, C. De Nisco, M. De Prizio, A. De Sol, A. Dibella, T. Falcioni, N. Falco, C. Farina, E. Finotti, T. Fontana, G. Francioni, P. Fransvea, B. Frezza, G. Garbarino, G. Garulli, M. Genna, S. Giannessi, A. Gioffrè, A. Giordano, D. Gozzo, S. Grimaldi, G. Gulotta, V. Iacopini, T. Iarussi, G. Laracca, E. Laterza, A. Leonardi, L. Lepre, L. Lorenzon, G. Luridiana, A. Malagnino, G. Mar, P. Marini, R. Marzaioli, G. Massa, V. Mecarelli, P. Mercantini, A. Mingoli, G. Nigri, S. Occhionorelli, N. Paderno, GM. Palini, D. Paradies, M. Paroli, F. Perrone, N. Petrucciani, L. Petruzzelli, A. Pezzolla, D. Piazza, V. Piazza, M. Piccoli, A. Pisanu, M. Podda, G. Poillucci, R. Porfidia, G. Rossi, P. Ruscelli, A. Spagnoli, R. Sulis, D. Tartaglia, C. Tranà, A. Travaglino, P. Tomaiuolo, A. Valeri, G. Vasquez, M. Zago, E. Zanoni.

Funding None. 
Data availability Data are available on request to the corresponding author.

\section{Compliance with ethical standards}

Conflict of interest All the authors declare to have no conflict of interest.

Ethical approval The protocol of the present study was approved by the ethical committee of the Sapienza University, Rome, Italy (Prot. n. 231 SA_2016 del 12.12.2016).

Consent to participate All the patients approved to participate to the study signing a specific form after careful information.

Consent for publication All the patients approved the publication of the study results.

Open Access This article is licensed under a Creative Commons Attribution 4.0 International License, which permits use, sharing, adaptation, distribution and reproduction in any medium or format, as long as you give appropriate credit to the original author(s) and the source, provide a link to the Creative Commons licence, and indicate if changes were made. The images or other third party material in this article are included in the article's Creative Commons licence, unless indicated otherwise in a credit line to the material. If material is not included in the article's Creative Commons licence and your intended use is not permitted by statutory regulation or exceeds the permitted use, you will need to obtain permission directly from the copyright holder. To view a copy of this licence, visit http://creativecommons.org/licenses/by/4.0/.

\section{References}

1. HerniaSurge Group (2018) International guidelines for groin hernia management. Hernia 22:1-165. https://doi.org/10.1007/s1002 9-017-1668-x

2. Compagna R, Rossi R, Fappiano F et al (2013) Emergency groin hernia repair: implications in elderly. BMC Surg 13(Suppl 2):S29. https://doi.org/10.1186/1471-2482-13-S2-S29

3. Simons MP, Aufenacker T, Bay-Nielsen M et al (2009) European Hernia Society guidelines on the treatment of inguinal hernia in adult patients. Hernia 13:343-403. https://doi.org/10.1007/s1002 9-009-0529-7

4. Gallegos NC, Dawson J, Jarvis M, Hobsley M (1991) Risk of strangulation in groin hernias. Br J Surg 78:1171-1173. https:// doi.org/10.1002/bjs. 1800781007

5. EU Hernia Trialists Collaboration (2002) Repair of groin hernia with synthetic mesh: meta-analysis of randomized controlled trials. Ann Surg 235:322-332. https://doi.org/10.1097/00000658200203000-00003

6. Kingsnorth A, LeBlanc K (2003) Hernias: inguinal and incisional. Lancet 362:1561-1571. https://doi.org/10.1016/S0140 -6736(03)14746-0

7. Turrentine FE, Wang H, Simpson VB, Jones RS (2006) Surgical risk factors, morbidity, and mortality in elderly patients. J Am Coll Surg 203:865-877. https://doi.org/10.1016/j.jamcollsur g.2006.08.026

8. Costa G, ERASO (Elderly Risk Assessment for Surgical Outcome) Collaborative Study Group, Massa G (2018) Frailty and emergency surgery in the elderly: protocol of a prospective, multicenter study in Italy for evaluating perioperative outcome
(The FRAILESEL Study). Updates Surg 70:97-104. https://doi. org/10.1007/s13304-018-0511-y

9. Charlson M, Szatrowski TP, Peterson J, Gold J (1994) Validation of a combined comorbidity index. J Clin Epidemiol 47:12452125. https://doi.org/10.1016/0895-4356(94)90129-5

10. Rashid M, Kwok CS, Gale CP et al (2017) Impact of co-morbid burden on mortality in patients with coronary heart disease, heart failure, and cerebrovascular accident: a systematic review and meta-analysis. Eur Heart J Qual Care Clin Outcomes 3:20-36. https://doi.org/10.1093/ehjqcco/qcw025

11. Yan J, Wang Y-X, Li Z-P (2011) Predictive value of the POSSUM, p-POSSUM, cr-POSSUM, APACHE II and ACPGBI scoring systems in colorectal cancer resection. J Int Med Res 39:1464-1473. https://doi.org/10.1177/147323001103900435

12. Echara ML, Singh A, Sharma G (2019) Risk-adjusted analysis of patients undergoing emergency laparotomy using POSSUM and P-POSSUM Score: a prospective study. Niger J Surg 25:45-51. https://doi.org/10.4103/njs.NJS_11_18

13. Borchardt RA, Tzizik D (2018) Update on surgical site infections: the new CDC guidelines. JAAPA 31:52-54. https://doi. org/10.1097/01.JAA.0000531052.82007.42

14. Fitzgibbons RJ, Giobbie-Hurder A, Gibbs JO et al (2006) Watchful waiting vs repair of inguinal hernia in minimally symptomatic men. JAMA 295:285. https://doi.org/10.1001/jama.295.3.285

15. O’Dwyer PJ, Chung L (2006) Watchful waiting was as safe as surgical repair for minimally symptomatic inguinal hernias. Evid Based Med 11:73. https://doi.org/10.1136/ebm.11.3.7

16. Birindelli A, Sartelli M, Di Saverio S et al (2017) 2017 update of the WSES guidelines for emergency repair of complicated abdominal wall hernias. World J Emerg Surg 12:37. https://doi. org/10.1186/s13017-017-0149-y

17. Kehlet H, Bay-Nielsen M, Collaboration DHD (2008) Nationwide quality improvement of groin hernia repair from the Danish Hernia Database of 87,840 patients from 1998 to 2005. Hernia 12:1-7. https://doi.org/10.1007/s10029-007-0285-5

18. Helgstrand F, Rosenberg J, Kehlet H, Bisgaard T (2013) Outcomes after emergency versus elective ventral hernia repair: a prospective nationwide study. World J Surg 37:2273-2279. https://doi. org/10.1007/s00268-013-2123-5

19. Pokorny H, Klingler A, Schmid T et al (2008) Recurrence and complications after laparoscopic versus open inguinal hernia repair: results of a prospective randomized multicenter trial. Hernia 12:385-389. https://doi.org/10.1007/s10029-008-0357-1

20. Nilsson H, Nilsson E, Angerås U, Nordin P (2011) Mortality after groin hernia surgery: delay of treatment and cause of death. Hernia 15:301-307. https://doi.org/10.1007/s10029-011-0782-4

21. Alvarez Pérez JA, Baldonedo RF, Bear IG et al (2003) Emergency hernia repairs in elderly patients. Int Surg 88:231-237

22. Dahlstrand U, Wollert S, Nordin P et al (2009) Emergency femoral hernia repair: a study based on a national register. Ann Surg 249:672-676. https://doi.org/10.1097/SLA.0b013e31819ed943

23. Bittner R, Sauerland S, Schmedt C-G (2005) Comparison of endoscopic techniques vs Shouldice and other open nonmesh techniques for inguinal hernia repair: a meta-analysis of randomized controlled trials. Surg Endosc 19:605-615. https://doi. org/10.1007/s00464-004-9049-9

24. Malik HA, Ghanshan, Mumtaz A, Bano F (2017) Postoperative pain in laparoscopic transabdominal preperitoneal versus Lichtenstein open mesh repair techniques for inguinal hernia. J Surg Pak 22(3):79-82. https://doi.org/10.21699/jsp.22.3.3

25. Zafar H, Zaidi M, Qadir I, Memon AA (2012) Emergency incisional hernia repair: a difficult problem waiting for a solution. Ann Surg Innov Res 6:1. https://doi.org/10.1186/1750-1164-6-1

26. Xourafas D, Lipsitz SR, Negro P et al (2010) Impact of mesh use on morbidity following ventral hernia repair with a simultaneous 
bowel resection. Arch Surg 145:739-744. https://doi.org/10.1001/ archsurg.2010.144

Publisher's Note Springer Nature remains neutral with regard to jurisdictional claims in published maps and institutional affiliations. 